\title{
A novel two-stage repair technique for the management of esophageal perforation
}

\author{
Juha Saarnio, MD, PhD, Heikki Wiik, MD, PhD, Vesa Koivukangas, MD, PhD, \\ Timo Heikkinen, MD, PhD, Tatu Juvonen, MD, PhD, and Fausto Biancari, MD, PhD, \\ Oulu, Finland
}

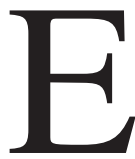
sophageal perforation is associated with significant mortality and morbidity. When primary surgical closure of the esophageal tear or its sealing by covered stents is not indicated, a 2-stage procedure with esophagectomy, end-cervical esophagostomy, and gastrostomy followed by reconstruction several months after mediastinitis subsides is considered the safest approach. ${ }^{1-3}$ However, preparation of the esophageal substitute and re-establishing esophageal continuity in a scarred posterior mediastinum poses further immediate and late risks to the patient. To reduce the extent of this second operative stage, we recently used a new method for 2-stage esophageal repair: a gastric tube was tunnelled via the posterior mediastinal route during the first procedure and a cervical gastrostomy along with a cervical esophagostomy was constructed. A simple cervical end-to-end esophagogastrostomy was then accomplished a few months later.

\section{Clinical Summaries}

PATIENT 1. A 41-year-old woman had esophageal and prepyloric gastric corrosive strictures after attempting suicide by ingesting natrium hydroxide. Two months later, after an endoscopic dilatation of an 11-cm long esophageal stricture, a 3-mm long perforation of the distal third of the thoracic esophagus developed. At emergency laparotomy, a markedly dilated stomach above a prepyloric corrosive stricture was found and a pyloro-prepyloroplasty was carried out. After resection of the intrathoracic esophagus and mediastinal débridement, a gastric tube was constructed by staplers and brought via the posterior mediastinal route to the neck. Because of the presence of prepyloric stricture that required surgical repair, we decided not to carry out a primary esophagogastrostomy. Thus, esophagostomy and gastrostomy to the neck and a feeding jejunostomy were constructed. The recovery was uneventful. Two and one-half months later, the pylorus and antrum were shown to be widely patent at a barium study performed through the cervical gastrostomy. The next day, an end-to-end esophagogastrostomy was accomplished. The recovery was uneventful and she was discharged on the ninth postoperative day. At a 1-month

From the Department of Surgery, Oulu University Hospital, Oulu, Finland. Received for publication Oct 24, 2006; accepted for publication Nov 28, 2006.

Address for reprints: Fausto Biancari, MD, PhD, Division of Cardiothoracic and Vascular Surgery, Department of Surgery, Oulu University Hospital, PO Box 21, 90029 Oulu, Finland (E-mail: faustobiancari@ yahoo.it; fausto.biancari@ppshp.fi).

J Thorac Cardiovasc Surg 2007;133:840-1

$0022-5223 / \$ 32.00$

Copyright (C) 2007 by The American Association for Thoracic Surgery doi:10.1016/j.jtcvs.2006.11.021 postoperative follow-up visit, she reported having vomited, which was found to be related

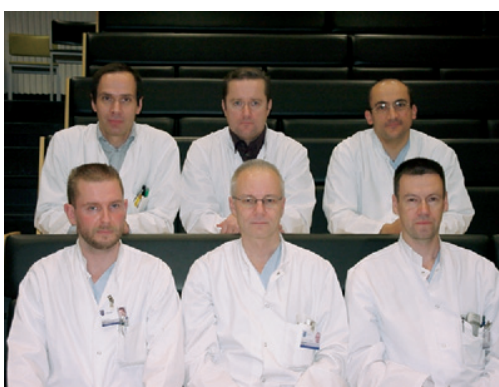

Drs Heikkinen, Juvonen, and Biancari (upper left to right); and Drs Koivukangas, Wiik, and Saarnio (lower left to right) to recurrent prepyloric stricture. An endoscopic dilatation of the pylorus and antrum was planned.

PATIENT 2. A 50-year-old man with alcoholism was admitted with a diagnosis of pneumonia. Because of worsening septic conditions, 2 days later a computed tomographic scan was done, and it showed leakage of contrast agent from the thoracic esophagus. At emergency laparotomy, a 5-cm long esophageal tear was found immediately above the gastroesophaeal junction. After resection of the intrathoracic esophagus and mediastinal débridement, a gastric tube was constructed by staplers and brought via the posterior mediastinal route to the neck. At this stage the distal part of the gastric tube was found to be slightly ischemic. Esophagostomy and gastrostomy as well as a feeding jejunostomy were constructed. Debridement and irrigation of the right pleura was accomplished through a thoracotomy. Eight days later, because of necrosis of the distal end of the gastric tube, $2 \mathrm{~cm}$ of the gastric tube was resected and a new gastrostomy to the neck was carried out. Intraoperative endoscopic examination confirmed that the remaining gastric tube was vital. Forty days after the operation, he was discharged. Six months later, a cervical esophagogastrostomy with 4-0 byosin was carried out. Postoperatively, a mild anastomotic leak occurred and rapidly healed. He was discharged on the 23rd postoperative day.

\section{Discussion}

Although more conservative approaches such as sealing with covered stents or primary closure of the esophageal tear are currently advocated, ${ }^{4,5}$ in a few patients, esophagectomy with diversion and late reconstruction remains the safest approach. The technique we have herein reported is meant to somewhat reduce the extent of this 2-stage surgical procedure by simplifying the second operative stage. Furthermore, it avoids the need for late colon interposition via the substernal route, which is a lengthy operation including the construction of three anastomoses; furthermore, the swallowing route is not direct and may cause graft angulation, and it may interfere with possible cardiac surgery. Our technique also allows the surveillance of the vitality of the esophageal substitute as well as of the cervical esophagus after the primary operation. It is easy to examine the gastric tube by endoscopy or barium study via cervical gastrostomy before any cervical anastomosis is accomplished. The second-stage procedure includes only a cervical end-to-end esophagogastrostomy anastomosis, which is 
a simple and not very costly operation with a relatively fast recovery.

In conclusion, the advantages of this 2-stage surgical technique are the reduction of the extent and costs of the second-stage operation along with the advantages of reconstruction with a gastric tube via the posterior mediastinum.

\section{References}

1. Kollmar O, Lindemann W, Richter S, Steffen I, Pistorius G, Schilling MK. Boerhaave's syndrome: primary repair vs. esophageal resectioncase reports and meta-analysis of the literature. J Gastrointest Surg. 2003;7:726-34
2. Tomaselli F, Maier A, Pinter H, Smolle-Juttner F. Management of iatrogenous esophagus perforation. Thorac Cardiovasc Surg. 2002;50: 168-73.

3. Salo JA, Isolauri JO, Heikkila LJ, Markkula HT, Heikkinen LO, Kivilaakso EO, et al. Management of delayed esophageal perforation with mediastinal sepsis: esophagectomy or primary repair? J Thorac Cardiovasc Surg. 1993;106:1088-91.

4. Fischer A, Thomusch O, Benz S, von Dobschuetz E, Baier P, Hopt UT. Non operative treatment of 15 benign esophageal perforations with self-expandable covered metal stents. Ann Thorac Surg. 2006;81:46773.

5. Vogel SB, Rout WR, Martin TD, Abbitt PL. Esophageal perforation in adults: aggressive, conservative treatment lowers morbidity and mortality. Ann Surg. 2005;241:1016-21.

\title{
Comparisons of infection complications between continuous flow and pulsatile flow left ventricular assist devices
}

\author{
Allison R. Schulman, BA, ${ }^{\text {a }}$ Timothy P. Martens, MD, ${ }^{a}$ Paul J. Christos, MPH, MS, ${ }^{\text {b }}$ Mark J. Russo, MD, MS, ${ }^{a}$ \\ George M. Comas, MD, ${ }^{a}$ Faisal H. Cheema, MD, ${ }^{a}$ Tariq M. Naseem, MD, ${ }^{a}$ Raymond Wang, BA, ${ }^{a}$ \\ Katharine A. Idrissi, MSN, ${ }^{a}$ Stephen H. Bailey, MD, ${ }^{a}$ and Yoshifumi Naka, MD, PhD, ${ }^{a}$ New York, NY
}

$\mathrm{T}$ he implantation of a left ventricular assist device (LVAD) both as a bridge to transplantation and as destination therapy is being used with increasing frequency in patients with end-stage heart failure. Two main types of LVADs are currently being used: pulsatile and continuous flow devices. Continuous devices are much smaller and produce a continuous flow with either an axial or centrifugal flow pump. These devices fill during both the systolic and diastolic phase. $^{1}$

Despite the overall success of LVAD support and the advances in design in both types of devices, infection continues to be a common morbidity of mechanical circulatory support and remains a serious threat to the long-term survival of patients using LVADs. ${ }^{2,3}$ This study was designed to determine the differences in infection rates between patients with puslatile pumps (HeartMate I; Thermo Cardiosystems, Inc, Woburn, Mass) versus those with axial flow devices (HeartMate II or DeBakey; MicroMed Technology, Inc, Houston, Tex).

From the Division of Cardiothoracic Surgery, Columbia University Medical Center, ${ }^{a}$ and the Division of Biostatistics and Epidemiology, Department of Public Health, Weill Medical College of Cornell University, ${ }^{\mathrm{b}}$ New York, NY.

Received for publication Sept 23, 2006; accepted for publication Sept 28, 2006.

Address for reprints: Yoshifumi Naka, MD, PhD, Division of Cardiothoracic Surgery, 177 Fourth Washington Ave, MHB 7-435, New York, NY 10032 (E-mail: yn33@columbia.edu).

J Thorac Cardiovasc Surg 2007;133:841-2

$0022-5223 / \$ 32.00$

Copyright $\odot 2007$ by The American Association for Thoracic Surgery doi:10.1016/j.jtcvs.2006.09.083

\section{Patients and Methods}

We retrospectively reviewed the case histories of 92 patients undergoing LVAD implantation at a single center between October 2003 and April 2006. This analysis captured the first 27 axial flow device implants at our center, as well as every HeartMate I device implanted during the same interval. LVADs were classified as pulsatile $(\mathrm{n}=65)$ or continuous flow $(\mathrm{n}=27)$. Local device infection was defined by clinical signs of infection with positive culture(s) from the abdominal wound, driveline, pocket, or pump. The $\chi^{2}$ test was used to analyze the associations between device type and categorical variables (device infections, gender, preoperative history of diabetes or hypertension, bridge-to-transplant rate, and post-implant 1-year survival), and the nonparametric MannWhitney test was used to analyze associations between device type and continuous variables (age, height, body surface area [BSA], and body mass index [BMI]).

\section{Results}

Findings are summarized in Table 1. There were no significant differences between pulsatile and continuous device groups with respect to age, gender, height, or preoperative history of diabetes or hypertension. BSA (2.0 vs $1.89 ; P=.035)$ and BMI (28.6 vs $24.9 ; P=.009)$ were significantly higher in patients with pulsatile devices. Patients with pulsatile devices were more likely to have an LVAD-related infection (18/65 vs $1 / 27,27.7 \%$ vs $3.7 \% ; P=$ $.010)$, in particular pocket infections $(10 / 65$ vs $0 / 27,15.4 \%$ vs $0 \%$; $P=.031)$ and wound infections ( $10 / 65$ vs $0 / 27,15.4 \%$ vs $0 \%$; $P=.031)$. Of note, the rates of driveline infection $(9 / 65 \mathrm{vs}$ $2 / 27,13.8 \%$ vs $7.4 \% ; P=.271)$ and pump endocarditis $(2 / 62 \mathrm{vs}$ $1 / 26,3.2 \%$ vs $3.7 \%$; $P=.999)$ were comparable between the two groups. Device infections had no effect on bridge-totransplant rate $(15 / 18$ vs $39 / 56,83.3 \%$ vs $69.6 \% ; P=.364)$ or 